\title{
Timing of Anticoagulant Therapy After Acute Ischemic Stroke
}

\author{
Ryu Matsuo, MD, PhD; Masahiro Kamouchi, MD, PhD
}

A nticoagulation is an effective means of preventing recurrent stroke in patients with ischemic stroke caused by nonvalvular atrial fibrillation (NVAF). ${ }^{1}$ Oral anticoagulants (OACs) reduce the risk of cerebrovascular accident and systemic embolism, despite the increased risk of bleeding. The use of OACs is strongly recommended for secondary prevention in patients with NVAF. Nevertheless, it is still unclear whether early initiation of OAC therapy is beneficial after acute ischemic stroke caused by NVAF.

The risk of stroke recurrence is high in the early stage of acute ischemic stroke. In patients with cardioembolic stroke, the rate of early recurrence of ischemic stroke, defined as a new event within 2 weeks of onset, is reportedly $1-10 \%{ }^{2} \mathrm{~A}$ meta-analysis showed that early initiation of low-molecular weight heparin (LMWH), unfractionated heparin (UFH) or heparinoid within $48 \mathrm{~h}$ of cardioembolic stroke was not associated with a reduced risk of recurrent ischemic stroke, death or disability, but did increase the risk of intracranial hemorrhage (ICH). ${ }^{3}$ The increased incidence of $\mathrm{ICH}$ negated any benefit of early treatment with anticoagulants for the prevention of recurrent ischemic events. ${ }^{4}$ Currently, OACs are generally initiated within 1-2 weeks of stroke onset, but the evidence informing this practice is limited. ${ }^{5}$ Current expert consensus is that earlier anticoagulation could be considered for patients at low risk of bleeding (e.g., those with a small infarct or without evidence of hemorrhagic transformation on brain imaging) ${ }^{6}$

Recent analysis of the Virtual International Stroke Trials Archive (VISTA) found that early introduction of anticoagulants 2-3 days after stroke was associated with substantially fewer recurrent strokes over the following weeks without excess risk of symptomatic ICH.7 Additionally, the Early Recurrence and Cerebral Bleeding in Patients With Acute Ischemic Stroke and Atrial Fibrillation (RAF) study showed that initiation of anticoagulation treatment between 4 and 14 days after acute ischemic stroke was safe and effective compared with starting treatment before or afterwards. ${ }^{8}$ Those results suggested that early initiation of anticoagulation therapy is beneficial in patients with acute ischemic stroke caused by NVAF. The most recent consensus recommendation on the timing of initiation of anticoagulation is that OACs can be initiated at $1,3,6$ or 12 days after onset, guided by stroke severity and considering the risk of hemorrhagic transformation. ${ }^{9}$

\begin{abstract}
Article p 180
As well as the timing of initiation, the choice of $\mathrm{OAC}$ is also critical. Vitamin K antagonists (VKAs) require a longer time to sufficiently inhibit coagulation than intravenous heparin. Therefore UFH, or preferably $\mathrm{LMWH}$, is often used as secondary prevention during the acute stage of cardioembolic stroke. The newer direct OACs (DOACs) have a more rapid onset of action than VKAs, and full anticoagulation can be achieved within hours of the first dose. Furthermore, DOACs cause fewer hemorrhagic complications than VKAs. Even though the incidence of ICH is higher in Asians, DOACs are reported to substantially decrease the incidence of ICH in Asian patients with NVAF compared with VKAs. ${ }^{10}$ Thus, DOACs may be useful as prophylactic anticoagulation in the early stages after ischemic stroke caused by NVAF. ${ }^{2}$

Seiffge et al11 recently assessed the starting time of DOACs for secondary prevention of stroke and the rate of
\end{abstract}
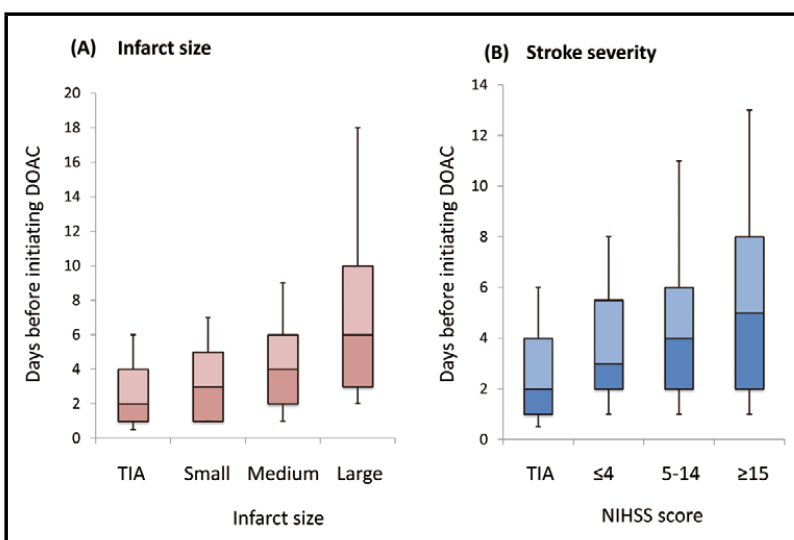

Figure. Number of days before initiating a direct oral anticoagulant according to infarct size $(\mathbf{A})$ and initial stroke severity assessed by NIHSS (B) in the Stroke Acute Management with Urgent Risk-factor Assessment and Improvement (SAMURAINVAF) registry. Boxes represent interquartile ranges, whiskers represent the 10th and 90th percentiles. NIHSS, National Institute of Health Stroke Scale; TIA, transient ischemic attack. (Reproduced with permission from Toyoda K, et al. ${ }^{13}$ )

The opinions expressed in this article are not necessarily those of the editors or of the Japanese Circulation Society.

Received December 14, 2016; accepted December 14, 2016; released online December 28, 2016

Department of Health Care Administration and Management (R.M., M.K.), Department of Medicine and Clinical Science (R.M.), Graduate School of Medical Sciences, Kyushu University, Fukuoka, Japan

Mailing address: Ryu Matsuo, MD, PhD, Department of Health Care Administration and Management, Graduate School of Medical Sciences, Kyushu University, 3-1-1 Maidashi, Higashi-ku, Fukuoka 812-8582, Japan. E-mail: rymatsuo@hcam.med. kyushu-u.ac.jp

ISSN-1346-9843 All rights are reserved to the Japanese Circulation Society. For permissions, please e-mail: cj@j-circ.or.jp 
$\mathrm{ICH}$ or recurrent ischemic events in 204 patients with acute ischemic stroke or transient ischemic attack and NVAF. They found that DOACs were started within 7 days in $65 \%$ of patients, but early initiation did not significantly influence the rate of recurrent stroke or ICH. Nomura et al12 evaluated the rates of new lesions and hemorrhagic transformation on diffusion-weighted magnetic resonance brain images in the first 2 weeks after acute ischemic stroke in patients with NVAF. They found no significant difference in the rates of new lesions $(26.0 \%$ vs. $28.0 \%)$ or hemorrhagic transformation $(30.0 \%$ vs. $39.2 \%)$ between 50 patients treated with DOACs and 125 patients treated with warfarin. In a multicenter prospective cohort study in Japan (the Stroke Acute Management with Urgent Risk-factor Assessment and Improvement [SAMURAI-NVAF] registry), DOACs were administered a median of 4 days after onset in patients with acute ischemic stroke and NVAF. The median time from onset to initiation of DOACs differed according to infarct size (transient ischemic attack, 2 days; small infarct, 3 days; medium infarct, 4 days; large infarct, 6 days) and the initial stroke severity (National Institute of Health Stroke Scale, NIHSS score $\leq 4,3$ days; NIHSS score 5-14, 4 days; NIHSS score $\geq 15,5$ days, Figure). ${ }^{13}$ There was no incidence of ICH in the patients taking DOACs. These results suggested that the risk of $\mathrm{ICH}$ was low even if DOACs were started soon after stroke onset.

In this issue of the Journal, Deguchi et $\mathrm{al}^{\mathbf{1 4}}$ report a retrospective analysis of the timing of OAC therapy in Japanese patients with acute ischemic stroke and NVAF. They found that the characteristics of the patients treated with DOACs differed from those treated with VKAs. Patients treated with DOACs were younger and had less severe strokes than those with VKAs. Furthermore, DOACs were started earlier than VKAs, even earlier than under the "1-3-6-12" rule. Also, no bleeding events were detected in the patients on DOACs, suggesting that early administration of a DOAC is safe in patients with ischemic stroke and NVAF. More data are needed to confirm the safety of DOACs administered in the hyper-acute stage of ischemic stroke.

Whether early administration of OACs is safe and effective is still unclear. To avoid a variety of potential biases underlying any observational study, further large-scale randomized controlled studies are needed in patients with acute ischemic stroke caused by NVAF. These should seek to establish the optimum initiation time for OACs to balance the prevention of recurrent stroke against the risk of $\mathrm{ICH}$, and whether DOACs are safer and more effective than other anticoagulants.

\section{References}

1. Secondary prevention in non-rheumatic atrial fibrillation after transient ischaemic attack or minor stroke. EAFT (European Atrial Fibrillation Trial) Study Group. Lancet 1993; 342: $1255-$ 1262.

2. Yamagami $\mathrm{H}$, Toyoda $\mathrm{K}$. Timing of anticoagulation therapy in patients with acute cardioembolic stroke. Circ J 2015; 79: 763 765.

3. Paciaroni M, Agnelli G, Micheli S, Caso V. Efficacy and safety of anticoagulant treatment in acute cardioembolic stroke: A meta-analysis of randomized controlled trials. Stroke 2007; 38: $423-430$.

4. Cucchiara B. How long should we wait to start oral anticoagulation after cardioembolic stroke? Neurology 2016; 87: 1852-1853.

5. Jauch EC, Saver JL, Adams HP Jr, Bruno A, Connors JJ, Demaerschalk BM, et al. Guidelines for the early management of patients with acute ischemic stroke: A guideline for healthcare professionals from the American Heart Association/American Stroke Association. Stroke 2013; 44: 870-947.

6. Lansberg MG, O'Donnell MJ, Khatri P, Lang ES, NguyenHuynh MN, Schwartz NE, et al. Antithrombotic and thrombolytic therapy for ischemic stroke: Antithrombotic Therapy and Prevention of Thrombosis, 9th ed: American College of Chest Physicians Evidence-Based Clinical Practice Guidelines. Chest 2012; 141: e601S-e636S

7. Abdul-Rahim AH, Fulton RL, Frank B, Tatlisumak T, Paciaroni M, Caso V, et al. Association of improved outcome in acute ischaemic stroke patients with atrial fibrillation who receive early antithrombotic therapy: Analysis from VISTA. Eur $J$ Neurol 2015; 22: $1048-1055$

8. Paciaroni M, Agnelli G, Falocci N, Caso V, Becattini C, Marcheselli S, et al. Early recurrence and cerebral bleeding in patients with acute ischemic stroke and atrial fibrillation: Effect of anticoagulation and its timing: The RAF Study. Stroke 2015; 46: $2175-2182$

9. Heidbuchel H, Verhamme P, Alings M, Antz M, Diener HC, Hacke W, et al. Updated European Heart Rhythm Association Practical Guide on the use of non-vitamin K antagonist anticoagulants in patients with non-valvular atrial fibrillation. Europace 2015; 17: 1467-1507.

10. Yasaka M, Lip GY. Impact of non-vitamin $\mathrm{k}$ antagonist oral anticoagulants on intracranial bleeding in Asian patients with non-valvular atrial fibrillation. Circ J 2014; 78: 2367-2372.

11. Seiffge DJ, Traenka C, Polymeris A, Hert L, Peters N, Lyrer P, et al. Early start of DOAC after ischemic stroke: Risk of intracranial hemorrhage and recurrent events. Neurology 2016; 87: $1856-1862$

12. Nomura A, Konno T, Fujita T, Tanaka Y, Nagata Y, Tsuda T, et al. Fragmented QRS predicts heart failure progression in patients with hypertrophic cardiomyopathy. Circ J 2015; 79: $136-143$.

13. Toyoda K, Arihiro S, Todo K, Yamagami H, Kimura K, Furui $\mathrm{E}$, et al. Trends in oral anticoagulant choice for acute stroke patients with nonvalvular atrial fibrillation in Japan: The SAMURAI-NVAF study. Int J Stroke 2015; 10: 836-842.

14. Deguchi I, Tanahashi N, Takao M. Timing of treatment initiation with oral anticoagulants for acute ischemic stroke patients with nonvalvular atrial fibrillation. Circ J 2017; 81: 180-184. 